\title{
Analysis on the Causes of Sports Injury in College Physical Education and Its Prevention Strategies
}

\author{
Sang Mengxin \\ Department of Foreign languages, Dongchang College of Liaocheng University, Shandong, China, \\ 252000
}

Keywords: college physical education; sports injury; cause; prevention strategy

\begin{abstract}
With the increasing demand of college students for physical exercise, the diversification and socialization of college sports activities, and the occurrence of sports injuries in sports is inevitable. If this attention is not enough, prevention is not effective, response measures are not timely and effective, will discourage students to sports activities, affect the normal learning and life. This paper first introduces the definition and classification of sports injury, then analyzes the causes of sports injury from different perspectives, and finally puts forward effective countermeasures to prevent sports injury, hoping to play a positive role in promoting the reduction of injury degree of college students in sports training.
\end{abstract}

\section{Introduction}

Physical training can strengthen students' physical quality and psychological quality. Physical training also has many advantages for students' life and study, but there is also a serious problem, that is, damage may occur during exercise. If the reason for the failure cannot make a correct judgment in time, then the correct recovery method cannot be adopted, and the sports injury of the college student cannot be prevented. Therefore, we must grasp the causes and laws of sports injuries. Take active and effective preventive measures to minimize the occurrence of sports injuries.

\section{Definition and classification of sports injuries}

The so-called sports injury refers to the anatomical destruction or physiological disorder of human tissues or organs in sports, which is called sports injury. Under normal circumstances, academic circles divide sports injuries into new injuries and old injuries according to time. According to the course of disease, they can be divided into acute injuries and chronic injuries. According to their nature, they can be divided into open injuries and closed injuries. According to the degree, they can be divided into mild and Moderate injury, serious injury ${ }^{[1]}$. The classification of sports injuries is shown in figure 1 . 


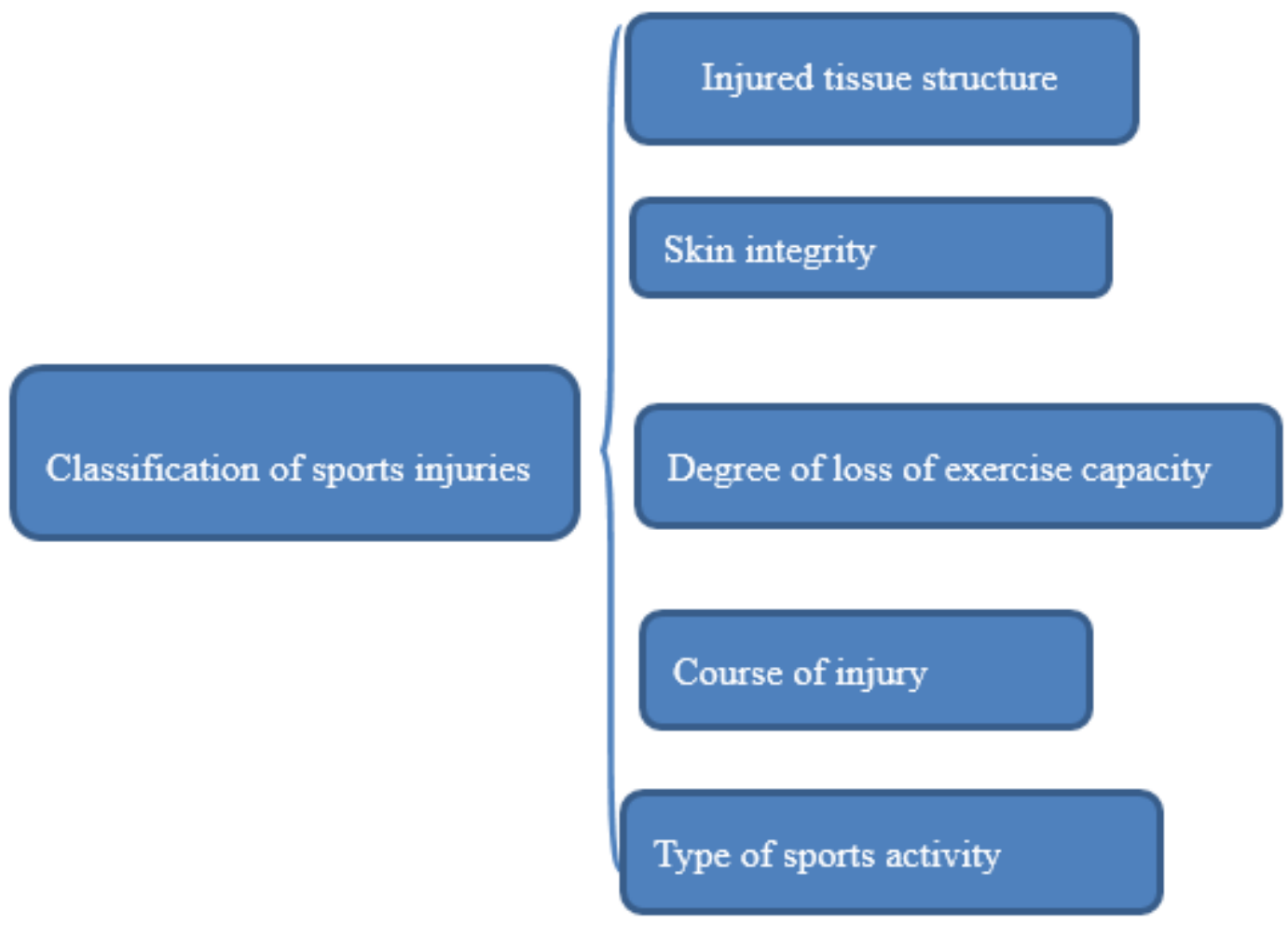

Figure1 Classification of sports injuries

\section{Analysis of the causes of sports injuries}

\subsection{Sports characteristics}

The causes of sports injuries are multiplicity and complexity. Ball games are the most vulnerable to sports injuries in all the more common sports. Table 2 is a survey of 220 college students who were injured in ball sports. Ball games have the largest number of injuries. This is because the ball project is characterized by direct contact with the body. Students have direct physical contact when stealing the ball in a tight and intense game. In basketball and football, the body can have a reasonable body. Collision, coupled with the savage student movement, deliberate fouls, do not play or exercise according to the rules, so there are more opportunities for injury ${ }^{[2]}$.

Table 2 College students' ball sports project questionnaire

\begin{tabular}{ccc}
\hline Ball sports & Number of injured & Proportion of injured \\
\hline football & 62 & $28.2 \%$ \\
basketball & 57 & $25.9 \%$ \\
badminton & 30 & $13.6 \%$ \\
volleyball & 66 & $30 \%$ \\
pingpong & 5 & $2.3 \%$ \\
\hline
\end{tabular}

\subsection{Basic body quality}

Excessive local load is the primary cause of injury. The outstanding problem among injured students is that their muscle strength is poor and their physical fitness is also low. If the amount of exercise during the competition or exercise is large, it will easily cause damage if it exceeds the range of the body's ability to withstand. Therefore, low physical fitness is an important factor in the 
occurrence of sports injuries.

\subsection{Impact of comprehensive objective factors}

There are many objective factors involved in the process of sports activities, especially outdoor sports activities, which have a direct relationship with weather, venues, sports equipment and other factors. The first is the weather factor. When college students are engaged in sports activities, they are too high or too low, which will lead to a significant reduction in the coordination of human body functions, which will affect the self-protection movements of college students, leading to the occurrence of sports injuries. In addition, the material conditions of sports venues in colleges and universities are also important influencing factors. The unscientific and imperfect nature of the venues will lead to the difficulty in the effective performance of the technical skills of college students in sports activities, and the resulting sports injuries. In addition, many college sports equipment is too old, lack of maintenance and renewal, which will become a potential danger in college students' sports activities ${ }^{[3]}$.

\subsection{Lack of self-protection}

The majority of sports injuries in sports practice activities are due to the insufficient attention of college students to sports safety. If you don't realize that danger may occur during the exercise, then the college students will not make protective actions, which will make it easy for the university to produce sports injuries. The lack of attention paid by college students to sports injuries is mainly due to the lack of safety knowledge of teachers in college physical education. In the process of sports, college students lack attention to sports safety awareness and sports safety. During the process of sports, they are not focused, laughing and laughing. This is the main cause of sports injuries in college students.

\subsection{Insufficient awareness and degree of preparation activities}

The purpose of preparation activities before sports activities is to gradually transition the human body from a quiet state to a state of exercise. The physiological role is to overcome the inertia of the body, to improve the excitability of the nervous system and the skill level of various organ systems, and to lay a solid foundation for formal activities. However, many college students do not pay enough attention to the meaning of preparation activities, and often do not do it seriously. This leaves a potential factor for the occurrence of sports injuries, so it is easy to be injured in sports. Not only that, but also the determinants of the full extent of the preparation activities, if the preparation is sufficient, then the degree of sports injuries is also very small.

\section{Prevention of sports injuries in college students' sports}

\subsection{Develop good sports habits}

The strict and sufficient preparation activities of college students before participating in sports are important measures to effectively reduce sports injuries. The technical essentials for pre-sports preparation activities are not difficult. Most college students are not prepared for activities due to insufficient attention to preparation activities or do not strictly enforce the standards of preparatory activities. Therefore, teachers should explain to students the importance of preparing activities and describe the specific application techniques for preparing activities. Through the guidance education of discourse, the college students can develop good sports habits, and then enable college students 
to consciously generate self-protection awareness and independently carry out preparatory activities before sports. The content of the preparation activities should be determined according to the content of the formal exercise, the physical condition of the individual and the weather conditions.

\subsection{Strengthen sports safety education}

Sports safety education focuses on cultivating college students' sports safety awareness, and teaches college students sports safety knowledge, so that college students can effectively use sports safety knowledge and skills to protect themselves and others in the process of sports. The teaching process of sports safety education can be carried out in coordination with the theory of sports professional knowledge. When the teacher teaches a certain sports technique to the university students, the physical knowledge of the sports action is also transmitted to the university students. In turn, college students can more fully understand the application of sports safety knowledge. In the practice training of college students in sports skills, teachers should maintain observations of college students and provide timely safety guidance for some dangerous actions. In addition, teachers should focus on cultivating college students' safety awareness so that college students can consciously pay attention to self-protection in sports ${ }^{[4]}$.

\subsection{Reasonably arrange exercise load}

The current college physical education teaching activities in China focus on cultivating college students' sports literacy and physical quality, and lay a foundation for the formation of college students' comprehensive quality. In the physical education activities of colleges and universities, sports that are more confrontational should be reduced or avoided, the competitive nature of sports should be weakened, and the spirituality and fitness of sports should be emphasized. Therefore, in the sports activities of colleges and universities, the load of sports should be reduced, and the amount of exercise load should be maintained to maintain fitness needs. Reasonable arrangement of exercise load can not only ensure the safety of college students' sports, but also meet the teaching requirements of exercising students' physical fitness. In the arrangement of sports activities, teachers should treat children according to their age, gender, physical fitness, health status, sports level and characteristics of sports items, and arrange sports loads in a targeted and reasonable manner ${ }^{[5]}$.

\subsection{Strengthen medical supervision}

The medical supervision work of college sports is aimed at the safety guarantee mechanism of college students' sports activities. In order to prevent college students' sports injuries as the work goal, sports protection measures and methods are implemented from multiple levels. It is composed of professional medical staff and physical education teachers. It inspects sports facilities before sports activities, educates college students on sports safety knowledge, and supervises college students to complete sports preparation activities. During the development of sports activities, relevant medical equipment and medical supplies are prepared to cope with possible sports injuries.

\section{Conclusions}

Generally speaking, there are many reasons for sports injury in college physical education, which is also a weak link in school sports safety. From school leaders to PE teachers to students should fully realize the importance of safety education. In the process of sports, we must enhance our vigilance, try to avoid sports injuries, and choose sports according to the range of sports we can 
bear, so as not to give ourselves too much pressure and burden. Colleges and universities should also be on movement security increase the propaganda dynamics, make every student realize prepared to warm up before exercise, pay attention to protect themselves, in the movement after exercise massage to relieve, only pay attention to detail, to avoid serious injury, to guarantee the safety of the student movement, promote students to learn and grow.

\section{References}

[1] Li Yang. On the Causes and Prevention of Sports Injury in Physical Education and Training [J]. Sports Expo, 2011(13):150-150.

[2] Wu Feiyan. Characteristics and prevention of common sports injuries in physical education teaching in colleges and universities [J]. Journal of Ningxia Medical University, 2003, 25(5): 390-390.

[3] Luo Xueting. On the Prevention Mechanism and Management Strategy of Sports Injury in College Physical Education [J]. Sports Expo, 2011(19):159-159.

[4] Wang Changan. Research on Causes and Prevention Strategies of College Students' Sports Dance Injury [J]. Hubei Sports Science and Technology, 2017, 38(2): 149-151.

[5] Cui Jinyi. Cause Analysis and Preventive Countermeasures of Sports Injury in High School Sports Candidates Training [J]. China Science and Technology Expo, 2012(6):284-284. 